\title{
Radio Observations Of A Nearby Type IIP SN 2012aw
}

\author{
Naveen Yadav ${ }^{1}$, Alak Ray ${ }^{1}$, Sayan Chakraborti ${ }^{2}$, \\ Christopher Stockdale ${ }^{3}$, Poonam Chandra ${ }^{4}$, Randall Smith ${ }^{2}$, \\ Rupak Roy ${ }^{5}$, Vikram Dwarkadas ${ }^{6}$, Firoza Sutaria ${ }^{7}$, \\ Dave Pooley $^{8}$, Brijesh Kumar ${ }^{5}$ and Subhash Bose ${ }^{5}$ \\ ${ }^{1}$ Tata Institute of Fundamental Research, \\ Mumbai, India \\ email: naveen.phys@gmail .com \\ ${ }^{2}$ Harvard-Smithsonian CfA, USA \\ ${ }^{3}$ Marquette University, USA \\ ${ }^{4}$ National Center for Radio Astronomy-TIFR, India \\ ${ }^{5}$ Aryabhhata Research Institute of Observational Sciences, India \\ ${ }^{6}$ University of Chicago, USA \\ ${ }^{7}$ Indian Institute of Astrophysics, India \\ ${ }^{8}$ Sam Houston State University, Huntsville, USA
}

\begin{abstract}
SN 2012AW is a type-IIP supernova which exploded in M95. In this paper we discuss the radio observations of this supernova and model them to determine the important parameters relevant to the explosion and the evolution of blast wave. We also determine the dominant cooling process important to this source.
\end{abstract}

Keywords: Radio continuum: general, Supernovae: individual (SN 2012aw), Methods: data analysis, Techniques: image processing, interferometric

\section{Introduction}

SN 2012aw is a bright type II-P supernova which exploded in the galaxy M95 (d 10 $\mathrm{Mpc}$ ). Spectra taken $4-5$ days after discovery showed it to be a type IIP explosion (Fagotti et al. (2012)). Fraser et al.identified a candidate progenitor in archival HST images. Fraser et al. (2012) and have inferred a progenitor mass in range $14-26 \mathrm{M}_{\odot}$, whereas Van Dyk et al. (2012) inferred a progenitor mass in range $17-18 \mathrm{M}_{\odot}$. Its progenitor seems to be a faint red supergiant. SN 2012aw is being extensively studied from optical to X-ray bands. We followed the object at radio wavelengths using JVLA and GMRT, targeting it at $L, C, S, X, K \mathscr{G} K a$ bands at multiple epochs.

\section{Radio Observations, Reduction \& Modeling}

SN 2012aw was first detected in radio JVLA- $K$ band $\sim 10$ days by Stockdale et al. (2012). We conducted EVLA and GMRT observations of 2012aw at various epochs extending up to 187 days after the explosion. These observations have been reduced using Astronomical Image Processing Software $(A I P S)$ standard techniques. Interferometric visibilities have been calibrated using standard calibrators. The single source data has been extracted using AIPS task SPLIT and imaged using IMAGR.

The radio emission can be modeled as synchrotron emission with a combination of absorption and cooling processes. It has an optically thin component and an optically thick 

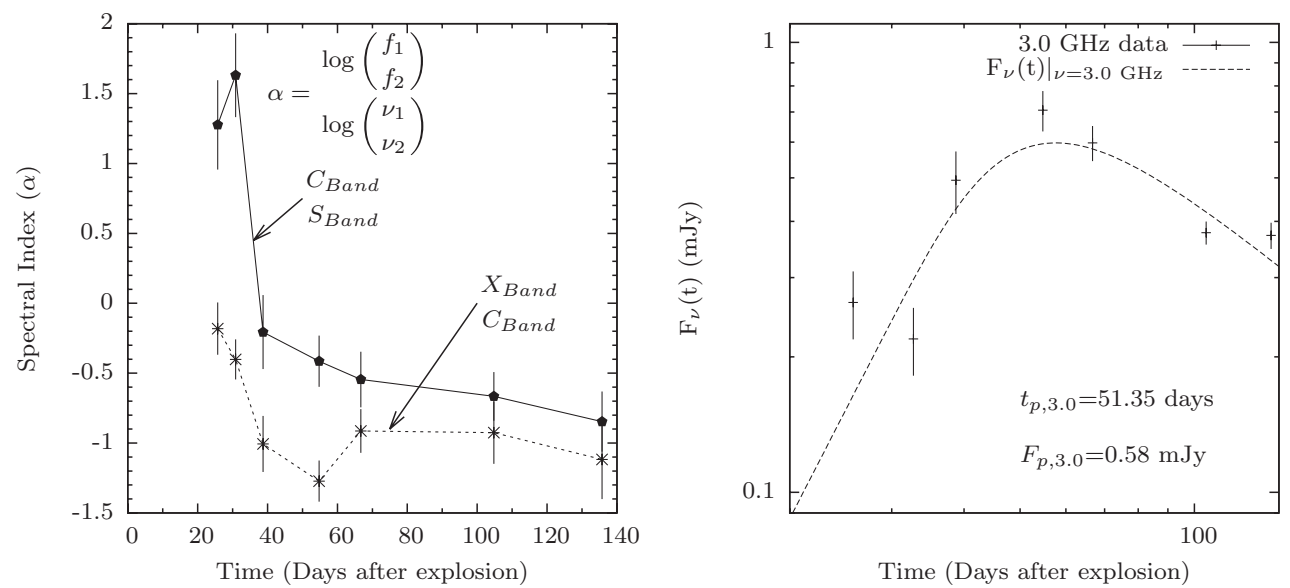

Figure 1. Left: Spectral index curves made using the $S, C \& X$ band data. Note the dip in the spectral index curve marked as $X_{B \text { and }} / C_{B \text { and }}$, is a signature of a cooling mechanism which was present at early time and is slowly turning off as the index approaches, -1 , its value in the optically thin regime. Right: An SSA fit to the $3.0 \mathrm{GHz}$ radio lightcurve using $\gamma=3.1$. It gives value of $t_{p, 3.0}=51.35$ days \& $F_{p, 3.0}=0.58 \mathrm{mJy}$.

component which can be modeled as a combination of synchrotron self absorption (SSA) $\&$ free-free absorption (FFA). We use Chevalier model-I (Chevalier (1996)) to study this emission. In this model the radius of interaction shell increases as, $R \propto t^{m}$ and energy density in relativistic electrons and magnetic fields also follows the hydrodynamical evolution with $u_{e}, u_{B} \propto t^{-2}$. In the analysis we assume that, $m \sim 1.0$. Electron index can be obtained by fitting a power law to the optically thin component. The equation for the radio flux evolution in such case of is given in Chevalier (1998) for the case of a supernova expanding in to a circumstellar medium set up by a uniform wind. In case of a source dominated by SSA the spectral index approaches value, $-(\gamma-1) / 2$, as the source enters the optically thin regime. If we try to model this object by simply using $S S A+F F A$, the fit gives $m$ greater than 1, implying an accelerated blast wave, which is incorrect as the blast wave decelerates due to its interaction with the circumstellar matter. Physically this can be explained as follows: the radio light curves of a source which is affected by a cooling mechanism (which slowly turns off) will be similar to a source which expands slowly at early times and the rate of expansion increases with time. This indicates the need to include a cooling mechanism which is dominant at early times and turns off at later times. In Figure 1(Left) the spectral index curve shows a sign of a cooling mechanism. We need to compare the cooling timescales for various mechanisms. The cooling timescales for an electron of energy $E$ can be written using formulas for energy loss from Pacholczyk (1970) as $t_{\text {Compton }}^{-1}=3.97 \times 10^{-2} u_{\text {rad }} E$ and $t_{\text {sync }}^{-1}=5.95 \times 10^{-2} u_{B} E$.

To get the synchrotron cooling timescale we need an estimate of magnetic field. In Chevalier model-I the magnetic field evolves as $t^{-1}$. If we know the magnetic field at one epoch it can simply be scaled to get the field at any other epoch using, $B(t)=B_{0}\left(t / t_{0}\right)^{-1}$. To get the value of magnetic field we can either use a late time radio spectrum or a low frequency radio lightcurve which are free from the electron cooling effects. To get an estimate of the FFA we use $\dot{M}_{-5} / v_{w 1}$ determined from time of X-ray detection. This object was first detected in X-ray $(0.2-10 \mathrm{KeV}$ band) by Immler et al. (2012) approximately 4 days after the explosion. This can be used to get an upper limit on the quantity $\dot{M}_{-5} / v_{w 1}$ which describes mass loss by a uniform wind. Using $t_{X}=4$ days, $v_{s 4} \sim 1.0$ and $E_{K e V}=1.0$ and adopting the value $C_{5}=2.6 \times 10^{6}$ in to the Equation 2.17 

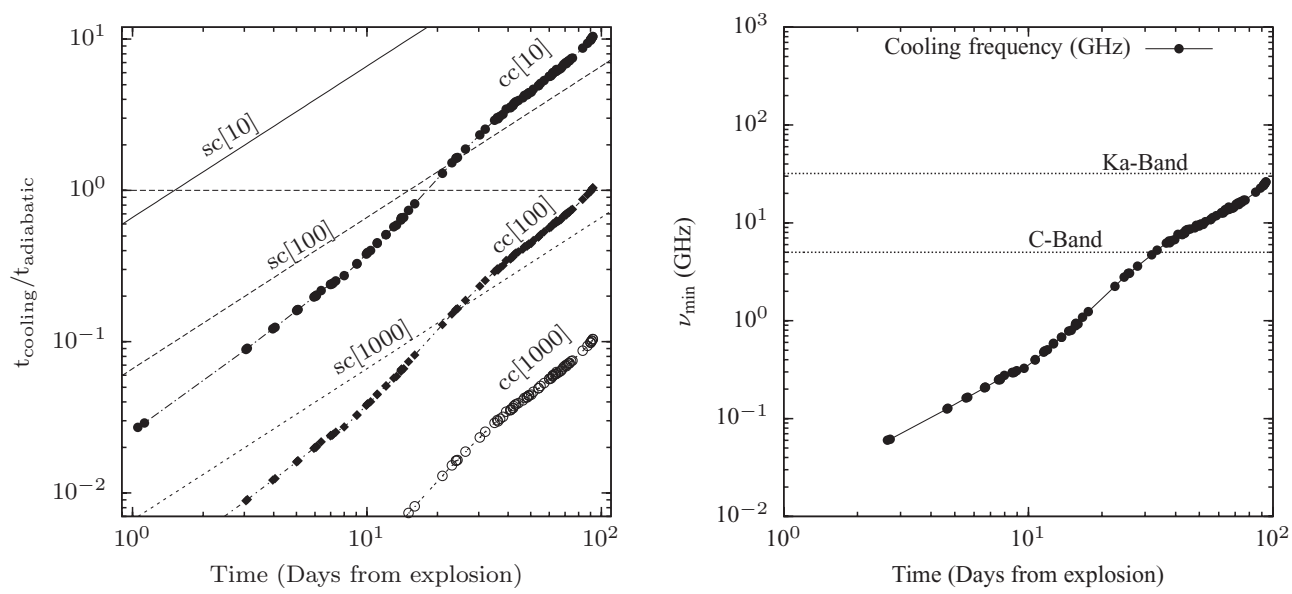

Figure 2. Left: Cooling timescales for electrons calculated using the computed bolometric light curve for various values of Lorentz factors as marked in the diagram by $\operatorname{sc}\left[\gamma_{i}\right]$ for synchrotron Cooling \& $\operatorname{cc}\left[\gamma_{i}\right]$ for Compton cooling respectively. Note that Compton cooling process is dominant over the synchrotron cooling process at any given Lorentz factor $\gamma_{i}$. Right: The minimum frequency affected by cooling plotted as a function of the age of SN 2012aw. Note that the dotted lines at $32.0 \mathrm{GHz}$ and $5.0 \mathrm{GHz}$ show that at early time all the VLA bands will have some effect of electron cooling. For the C-Band the cooling phase lasts till $\sim 30$ days.

from Chevalier et al. (1994), we get, $\dot{M}_{-5} / v_{w 1}<8.64 \times 10^{4} t_{X} v_{s 4} C_{5}^{-1} E_{K e V}^{8 / 3}<0.13$. where $\dot{M}_{-5}$ is mass loss rate in units of $10^{-5} \mathrm{M}_{\odot}, v_{w 1}$ is wind velocity in units of $10 \mathrm{Km} / \mathrm{s}, v_{s 4}$ is outer shock velocity in units of $10^{4} \mathrm{Km} / \mathrm{s}$ and $t_{X}$ is the time at which the medium becomes optically thin to X-rays of energy $E_{K e V}$ and $C_{5}$ is a constant. This value can be used to get an upper limit on the time for which free-free absorption dominates. Using Equation 4 from Chevalier et al. (2006) we get, $t_{f f} \approx 6\left(\dot{M}_{-6} / v_{w 1}\right)^{2 / 3} T_{c s 5}^{-1 / 2} v_{s 4}^{-1}(\nu / 8.46 \mathrm{GHz})^{-2 / 3}$. which gives $t_{f f} \leqslant 16.0$ days at $3.0 \mathrm{GHz}$ and $t_{f f} \leqslant 11.0$ days at $5.0 \mathrm{GHz}$ for $T_{c s 5}=1.0$. This shows that this object is not dominated by free-free absorption in $3.0 \mathrm{GHz}$ at early times. The $3.0 \mathrm{GHz}$ light curve can be fitted by a simple SSA model with a value of $m \sim 1.0$ as shown in Figure 1 (right). This can be used to derive the value of radius and magnetic field strength. Using the Equation 11 \& 12 from Chevalier (1998) gives $B_{0} \sim 0.48$ Gauss and $R_{0} \sim 3.9 \times 10^{15} \mathrm{~cm}$ on $\sim 51$ days assuming equipartition. We require the bolometric luminosity to determine the Compton cooling timescale. We construct a bolometric lightcurve using published photometric data from Bayless et al. (2013) \& Munari et al. (2013).

The calculated cooling timescales are shown in the Figure 2 (left). It is evident that for electrons of any given Lorentz factor $\gamma_{i}$ Compton cooling dominates over the synchrotron cooling mechanism. Therefore in order to model the radio spectrum at early epochs and at high frequency we need to consider the effect of cooling mechanism on emission. Assuming that the an electron emits synchrotron radiation at its characteristic frequency we can get an estimate of frequencies which are affected at a given age. Using $\nu_{c} \sim c_{1} B E^{2}$, where $c_{1}$ is a constant, the minimum frequency above which there are significant effects due to Compton cooling can be written as, $\nu_{\min }>0.78(t / 10$ days $)\left(L_{B o l} / 10^{42}\right)^{-2} \mathrm{GHz}$. The minimum frequency which is affected by cooling is shown in Figure 2 (right). It shows that at early times all the radio band is affected by cooling, but as the supernova fades $\nu_{\text {min }}$ goes to larger and larger values because of a decrease in bolometric luminosity and 
an increase in the radius of forward shock. As a result the cooling is important only for the highest frequencies at late times.

\section{Conclusions}

We discuss the radio observations of SN 2012aw. We study the rate at which the relativistic electrons at the radiosphere lose energy due to inverse Compton process and synchrotron process. We find that in the case of SN 2012aw Compton cooling dominates over the synchrotron cooling process. Therefore we note that Compton cooling effects need to be considered at early times in order to do a consistent modeling of the high frequency radio emission. This may help us to probe the particle acceleration process at the forward shock in a young radio bright supernova. In order to to gain a better understanding of the importance of cooling mechanisms we need to follow bright type II-P supernova as quickly as possible after type classification in radio and X-ray wavelengths.

\section{Acknowledgements}

The National Radio Astronomy Observatory is a facility of the National Science Foundation operated under cooperative agreement by Associated Universities, Inc. We would like to thank the Director EVLA for granting us DDT time on this object. One of the author Naveen Yadav wishes to acknowledge the support of CSIR-SPM fellowship.

\section{References}

Chevalier, R. \& Fransson, C. 1994, ApJ, 420, 268

Chevalier, R. 1998, ASP-CS, 93, 125

Chevalier, R. 1998, ApJ, 499, 810

Chevalier, R. \& Fransson, C. 2006, ApJ, 641, 1029

Fraser, M., Maund, J. R., Smartt, S. J., et al. 2012, Ap. Lett., 759, 13

Van Dyk, S. D., Cenko, S. B., Poznanski, D., et al. 2012, ApJ, 756, 131

Stockdale, C. J., Ryder, S. D., Van Dyk, S. D., et al. 2012, ATEL, 4012, 1

Yadav, N., Chakraborti, S., \& Ray, A. 2012, ATEL, 4010, 1

Fagotti, P., Dimai, A., Quadri, U., Strabla, L., et al. 2012, CBET, 3054, 1

Immler, S. \& Brown, P. J. 2012, ATEL, 3995, 1

Pacholczyk, A. G. 1970, Radio Astrophysics, San Francisco: Freeman, 1970

Munari, U., Henden, A., Belligoli, R., et al. 2013, New Astron., 20, 30

Bayless, A. J., Pritchard, T. A., et al. 2013, Ap. Lett., 764, L13

\section{Discussion}

Poonam Chandra: The formula for free-free absorption is steeper than a power law, but you seem to have used a power law form for FFA?

NAVEen YADAV: The actual formula used in the fitting has the exponential dependence (Eqn.9 from Chevalier (1998)).

Roger Chevalier: Your best fit has an $m>1.0$, which is not possible in the CSM interaction model?

NAVEEN YADAV: I agree that $m>1$ is unphysical, and have addressed the issue above. 